\title{
Multiple-unit activity of the rabbit medial geniculate nucleus in conditioning, extinction, and reversal
}

\author{
MICHAEL GABRIEL, JOSEPH D. MILLER, and STEVEN E. SALTWICK \\ University of Texas at Austin, Austin, Texas 78712
}

\begin{abstract}
Multiple-unit activity was recorded from the rabbit medial geniculate nucleus (MGN) during pretraining, avoidance conditioning, and extinction (Experiment 1), and during differential avoidance conditioning and reversal (Experiment 2). The CSs were tones and the UCS was shock in both studies. Locomotion within a wheel apparatus was the response. Three of the seven neuronal regions sampled in Experiment 1 showed evidence of short latency associative activity in the form of stimulus control during extinction. Five of seven regions sampled in Experiment 2 showed short-latency stimulus control, which developed during differential conditioning and reversed when the CS+ and CS- were interchanged.
\end{abstract}

An important issue in physiological psychology has been the question of whether or not primary sensory neural activity varies with change in the significance of incoming stimuli. Does a meaningful stimulus such as a signal for an aversive event produce different effects within the afferent pathway, relative to the effects of a physically similar, but neutral, stimulus?

The traditional view of the function of the primary sensory systems answers this question negatively. The principal function assumed by this view for sensory systems has been that of static "representation" of the outside world within the brain. This conception implies a constant sensory response to a constant stimulus, independent of the organism's state of attention or learning. Studies showing robustness and invariance of sensory evoked activity have supported this viewpoint. In these studies, sensory activity has maintained constancy across stages of sleep (Berlucci, Munson, \& Rizzolatti, 1967; Teas \& Kiang, 1964), whether or not anesthetics were present (Mori, Winters, \& Spooner, 1968) and whether the evoking stimuli were novel or familiar (Dunlop, Webster, \& Simon, 1965; Webster, 1971). In contrast, evoked neural activity of CNS systems other than the sensory systems has frequently shown covariation with sleep stage (e.g., Huttenlocher, 1961), anesthetics (e.g., Ammassian, 1954), and stimulus repetition (e.g., Horn \& Hill, 1964). Finally, studies of receptive-field properties of neurons in the visual system (Hubel \& Wiesel, 1959) have fostered a conception of fixed sensitivity to specific features of visual stimuli.

This research was supported by NIMH Grant MH 26276-01 and by grants from the Spencer Foundation and the Biomedical Sciences Committee. The authors wish to thank Linda Gabriel for her assistance in all phases of the research. Requests for reprints should be addressed to the first author.
Other viewpoints favor the idea that primary sensory activity changes in accordance with the significance of stimuli. For example, a variety of behavioral models are predicated on variation of sensory activity with attention to stimuli and stimulus meaning (e.g., Broadbent \& Gregory, 1964; Treisman, 1960). Moreover, the demonstrations of centrifugal pathways mediating modulatory effects at peripheral levels of some sensory systems (e.g., Granit, 1955; Watanabe, Yanagisawa, Kanzaki, \& Katsuki, 1966; Winter, 1965) provide a neuroanatomical basis for central control of incoming activity, and hence, a possible influence upon stimulus input by attention and learning. Finally, sensory modifiability is suggested by studies which have shown change in sensory evoked activity during repetitive (habituatory) stimulation, (e.g., Imig \& Weinberger, 1973; Simons, Dunlop, Webster, \& Aitkin, 1966; Wickelgren, 1968) and during behavioral conditioning (e.g., Buchwald, Halas, \& Schramm, 1966; Hall \& Mark, 1967)

Despite these areas of support, the idea of sensory modifiability is not one whose acceptance is compelled by empirical evidence. The behavioral models and the centrifugal modulatory effects are consistent with sensory modifiability, but not directly supportive of it. Studies employing repetitive stimulation have shown decrements in the afferent neural response, but they have not demonstrated a sufficient number of the other properties characteristic of behavioral response habituation (see Thompson \& Spencer, 1966). Thus, it remains unclear whether the phenomena reported in these studies represented instances of habituation, or whether, instead, the observed decrements may have reflected an unrecognized form of neural lability.

Finally, the studies of sensory evoked activity in conditioning have not provided clear demonstrations 
of associative change. Instead, the changes observed in the latter studies may have been produced by nonassociative factors such as general arousal and/or pseudoconditioning. Adequate controls for such factors were not employed.

In the present study, we attempted to demonstrate associative change of primary sensory (auditory) neuronal activity. As in other studies, conditioning was the basic method used to manipulate the significance of inputs to the auditory pathway. However, we attempted to circumvent the control problems of the past studies by use of conditioning procedures (stimulus generalization, differential conditioning, reversal) which have yielded unambiguously associative effects in behavioral studies. A preliminary report of the initial results of this approach is available elsewhere (Gabriel, Saltwick, \& Miller, 1975).

\section{METHODS}

\section{Subjects}

The data of the present report were obtained from 14 male albino rabbits. All of the rabbits were obtained from a local supplier. They weighed $1.5-2.0 \mathrm{~kg}$ at the time of their delivery to the laboratory, and were maintained on ad-lib food and water throughout the experiments.

\section{Procedure}

Electrodes and surgery. Surgical implantation of electrodes was carried out after a minimum period of $48 \mathrm{~h}$ of adaptation to laboratory conditions. Stainless steel electrodes made from No. 00 insect pins were stereotaxically implanted in the medial geniculate nucleus (MGN) while the rabbits were anesthetized. The electrode tips ranged in length from 20 to 60 microns. Multiple-unit activity was monitored continuously with an audio system and an oscilloscope, as an aid to electrode placement during implantation.

Recording and data analysis. The basic behavioral treatment was avoidance conditioning with a tone CS and a shock UCS. Throughout all procedures of conditioning, neuronal activity was fed through an amplifier (bandwidth $=500-10,000 \mathrm{~Hz}$ ) with a field effect transistor input. The output was recorded on standard magnetic tape. The details of the implantation procedure and of the recording system are provided in Gabriel (1973).

The tapes containing the neuronal records obtained during conditioning were played through high-pass filters and into Schmidt triggers. The trigger levels were set at a positive voltage level independently for each record. The specific setting was adopted such that only three or four of the largest neuronal spikes on each record contributed to the analysis. Records were discarded if they required a trigger setting below $+8 \mu \mathrm{V}$ to achieve this criterion.

The outputs of the Schmidt triggers (one pulse for each spike exceeding the trigger level) were fed into a PDP-12 computer programmed to count the number of pulses occurring in consecutive 10-msec periods ("bins") prior to and following the onset of the tone stimuli used for conditioning. The counts in each bin were summed over stimulus presentations (trials), resulting in a peristimulus histogram reflecting the frequency of multiple unit activity prior to and following tone onset. In all cases, the neuronal histograms constructed during behavioral training were based on 60 presentations of a stimulus.

Two types of information were obtained from the histograms. In order to evaluate neuronal activity present just after onset of the stimulus, the average of the frequencies in 30 bins just prior to stimulus onset $(\bar{X})$ was subtracted from the frequency $(X)$ in each of the initial 20 bins following stimulus onset. Then a " $Z$ '" score was computed using the standard deviation (S) of the 30 prestimulus bins. Thus $Z=(X-\bar{X}) / S$. Each $Z$ score reflected the frequency of neuronal firing in one of the 20 consecutive $10-\mathrm{msec}$ bins following stimulus onset. These scores may be referred to the $Z$ distribution to obtain a probability level, and a value of 1.96 represents the minimum $\mathrm{Z}$ required for statistical significance (two-tailed test, $\mathrm{p}<.05$ ).

The second method was designed to evaluate the amount of neuronal activity over a relatively gross interval $(600 \mathrm{msec})$ following onset of the stimulus. To achieve this, the frequencies accumulated in 10 consecutive $10-\mathrm{msec}$ bins were averaged to produce a single mean. Six such means were computed, representing six consecutive "blocks" of 10 bins each. A prestimulus mean based on 10 bins just prior to stimulus onset was subtracted from each of the six poststimulus means, and these differences were each divided by a standard error based on the prestimulus mean. Each of these scores reflected the magnitude of neuronal response in each of six consecutive 100 -msec periods following stimulus onset. These scores may be referred to the $t$ distribution to obtain a level of significance. A value of 2.1 represents the minimum $t$ required for statistical significance $(\mathrm{df}=18$, $\mathrm{p}<.05$, two-tailed test).

Histology. Following completion of behavioral testing, each rabbit was given an overdose of barbiturate, and perfused with saline, followed by $10 \%$ Formalin. Each brain was frozen and sectioned at 40 microns, and the sections containing the electrode tracks were photographed while still wet (Fox \& Eichman, 1959).

General behavioral procedure. Conditioning was carried out in a wheel apparatus (Brogden \& Culler, 1936), located within a shielding chamber, in a room adjacent to that containing the control apparatus. An exhaust fan and a speaker within the chamber produced a masking noise of $70 \mathrm{~dB}$ re $.0002 \mathrm{dyne} / \mathrm{cm}^{2}$, which was present throughout the experiments. The stimuli used as CSs and test stimuli in conditioning were pure tones $(8$ and $1 \mathrm{kHz}$ ), $80 \mathrm{~dB}$ re $.0002 \mathrm{dyne} / \mathrm{cm}^{2}$, with a rise time of $3 \mathrm{msec}$. The tones were played through a speaker located directly above the wheel. The UCS was a constant current shock $(1.5 \mathrm{~mA})$ delivered through the grid floor of the wheel, and a response was any wheel movement exceeding $.96 \mathrm{~cm}$ on the circumference of the wheel. During conditioning, the CS preceded the UCS by $5 \mathrm{sec}$, and both stimuli were response-terminated. A response in the presence of the CS prevented the occurrence of shock. The maximum duration of the combined CS and UCS, given failure of response to the UCS, was i sec. The interval between trials (ITI) was 10 , 15,20 , or $25 \mathrm{sec}$. These values occurred in a randomized sequence. A response during the ITI reset it. All daily sessions involved 120 trials with a single CS (conditioning procedure of Experiment 1), 60 with a CS and 60 with a non-CS tone (all other sessions). In the latter sessions, the tones were presented in a randomized order so that the subjects would not be able to predict which would occur, and therefore to orient differentially.

Pretraining. Each rabbit received pretraining, involving presentations of two tone stimuli ( 8 and $1 \mathrm{kHz}$ ), one of these to be a CS in conditioning and the other to be a generalization stimulus (Experiment 1, $\mathrm{n}=7$ ) or a CS - (Experiment 2, $\mathrm{n}=7$ ). Available evidence suggested equal sensitivity of the rabbit peripheral auditory mechanism to these frequencies (Price, 1963).

Two types of pretraining were used. One type involved presentations of the tones only and the other involved tones plus noncontingent presentations of the shock UCS, interspersed among the tones. Pretraining with shock involved an "explicitly unpaired" procedure (see Rescorla, 1967) in which the distribution of UCSs over trials was the same as the average distribution of UCSs given to 100 rabbits in past studies of conditioning (e.g., Gabriel, 1968). Unconditioned stimuli were given randomly within the ITI, with the restriction that no UCS occurred during the tone or within $3 \mathrm{sec}$ immediately preceding or following the tone. Three of the rabbits in Experiment 1 received nonshock pretraining and four received shock pretraining. Rabbits 
in Experiment 2 received both types of pretraining on 2 consecutive days, with order of treatments counterbalanced across subjects. Only the data of pretraining with shock will be reported in this paper. Details of the data resulting from both procedures of pretraining are provided in a separate report (Gabriel, Saltwick, \& Miller, Note 1).

Avoidance conditioning and overtraining (Experiment 1). After pretraining, consecutive sessions (days of avoidance conditioning, at 120 trials per day) were given until the first run of 10 consecutive avoidance CRs (criterion). Following criterion, 120 additional trials of overtraining were given in the same session $(n=4)$, or 120 trials in the same session plus two additional sessions of overtraining (120 trials each) were given on consecutive days $(\mathrm{n}=3) .{ }^{1}$ One of the tones used in pretraining was randomly selected to be the CS during conditioning and overtraining.

Extinction (Experiment 1). Following overtraining, 120 trials $(n=4)$ or 240 trials $(n=3)$ of extinction (UCS omitted) were given with two tones, half with the $\mathrm{CS}$ and half with the generalization tone. Three additional extinction sessions of 120 trials each were given on 3 consecutive days following the first extinction.

Differential conditioning and reversal (Experimental 2). Following pretraining, the rabbits in Experiment 2 received differential conditioning, i.e., conditioning as described above, but with two stimuli, CS + and CS -, each presented 60 times in each session. One of the tones used in pretraining was randomly designated $\mathrm{CS}+$, the other CS - . The UCS never followed the CS - . Differental conditioning was given daily to a criterion of two consecutive sessions in which $9 / 10$ responses to the $\mathrm{CS}+$ and 9/10 nonresponses to the CS - occurred in any 20-trial block. After attainment of criterion, each subject received additional sessions of "reversal," that is, the CS + and CS - were interchanged. Reversal was carried out to the same criterion as that used for differential conditioning. ${ }^{2}$ Each new procedure (e.g., differential conditioning) was always begun $24 \mathrm{~h}$ after the start of the previous procedure (e.g., pretraining).

\section{RESULTS}

\section{Experiment 1}

Behavioral responses. Figure 1 shows the mean frequency of the behavioral CRs of seven rabbits in pretraining, conditioning, overtraining, and extinction (Experiment 1).

Analysis of variance yielded a significant interaction of sessions and stimuli (CS vs. generalization tone) over the four consecutive sessions of extinction $(F=21.36$, df $=3 / 18 ; p<.001)$. This outcome, together with the results of subsequent individual comparisons, showed a greater mean frequency of behavioral CR to the CS compared to the generalization stimulus in the initial two sessions of extinction, but not in the final two sessions. That is, there was a significant "stimulus control" effect in the initial two sessions. Absence of a stimulus control effect in the third and fourth extinctions resulted from the very low frequency of responding to the stimuli in these sessions. The major point of interest was that the rabbits responded differentially to the cue (tone frequency) which was correlated with reinforcement.

In order to assess possible stimulus control of MGN activity, the $Z$ scores associated with the CS were compared to scores associated with the general-

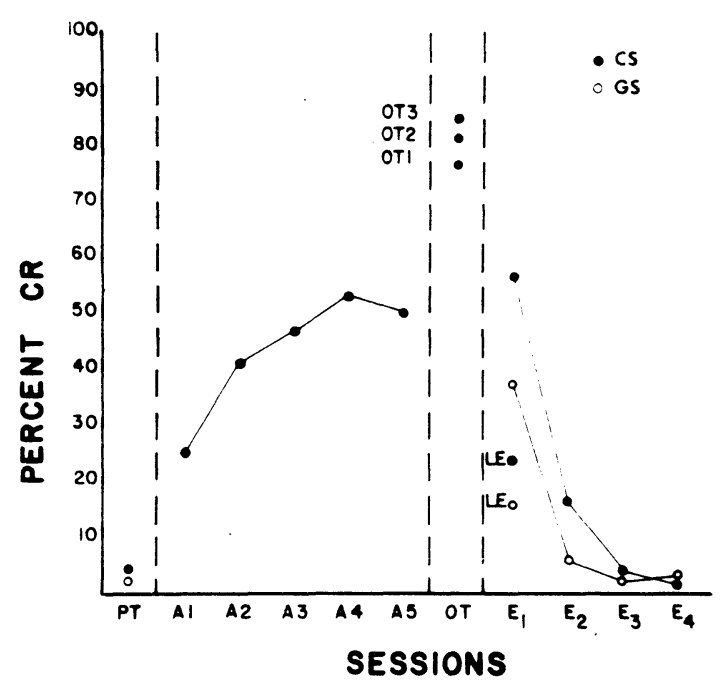

Figure 1. This graph shows the mean percentage of behavioral responses (percent $\mathrm{CR}$ ) to the $\mathrm{CS}(\bullet)$ and to the generalization stimulus (GS) $(O)$ over consecutive daily sessions. The data were obtained from the rabbits of Experiment 1. The pretraining (PT) points represent the average of shock and nonshock conditions. Percent CR for equal fifths of conditioning (excluding the criterial run) is graphed at abscissa points A1-A5. Data for the overtraining session are denoted OT1; percent $C R$ for animals receiving extended overtraining $(\mathrm{N}=3$ ) is denoted 0T2 (second session) and 0T3 (third session). The percent $C R$ shown at E1 represents the first 120 extinction trials of the rabbits who received extended extinction $(\mathbf{N}=3)$, combined with data from animals receiving standard 120 trial extinction. Percent $C R$ for the second 120 trials of extended extinction is labeled LE. Data for the final three extinction sessions are graphed at abscissa points E2-E4.

ization tone, in the four sessions of extinction, using the Wilcoxon matched pairs signed ranks test (Siegel, 1956). A significant stimulus control effect was our criterion of associative change in MGN. That is, a significantly greater neuronal response to the $\mathrm{CS}$, relative to the response to the generalization tone, was required in order to classify the activity of MGN as associative. This outcome was required in at least two of the four sessions of extinction. Prior to this analysis, the $\mathrm{Z}$ scores were corrected for possible prewired differential responses to the tones. This was done by subtracting the pretraining score for a given tone from the corresponding extinction score. Thus, significant differences observed during extinction between the neuronal responses to the tones existed after elimination of pretraining differences, and presumably were due to the interpolated conditioning treatment.

The neuronal responses ( $\mathrm{Z}$ scores) associated with the first 11 bins following CS onset were plotted for three rabbits in Figure $2(28,29$, and 35$)$. Tone onset occurs at the onset of Bin 2 and the abscissa marks denote the midpoint of each bin. Each row of the figure shows the data of a single rabbit in pretraining, overtraining, and in three sessions of extinction (1, 


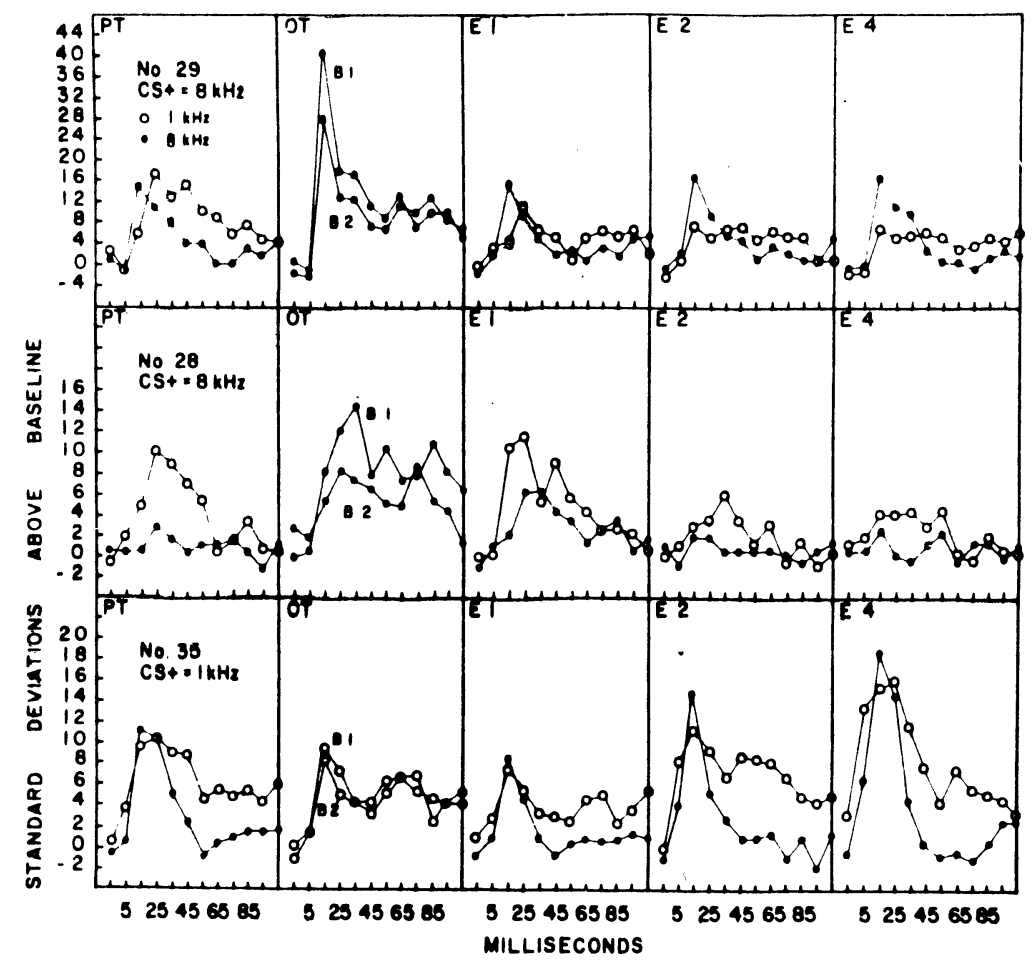

Figure 2. Each graph (e.g., left corner of Figure 2, 29) shows the neuronal response of the MGN in terms of $Z$ scores. Graphs are presented for noncontingent shock pretraining (PT), first overtraining session (OT), and first, second, and fourth extinction sessions (EXT1, EXT2, EXT4). Data based on the first 60 trials of $O T$ are labeled B1, and data based on the last 60 trials, B2. For rabbit 35, the first 120 trials of extinction were used to compute the $Z$ scores graphed in EXT1. Each graph spans 120 msec starting 10 msec prior to tone onset and including $110 \mathrm{msec}$ after tone onset. Tone onset in all graphs occurs at onset of bin 2 (second abscissa point). The abscissa marks denote the midpoint of each bin. In each graph, the neuronal response to the 8-kHz tone is denoted by filled circles (๑) and the response to the $1-\mathrm{kHz}$ tone by open circles $(O)$. The ordinate scale is in units of standard deviations from pretone baseline ( $Z$ scores). The abscissa of each graph represents time in milliseconds from tone onset to the midpoint of each 10-msec bin. Rabbits 28 and 29 received nonshock pretraining and rabbit 35 received shock pretraining.
2 , and 4). Three of the original seven rabbits $(24$, 28 , and 29) showed significant neuronal stimulus control in extinction. Neuronal response profiles of two of these are plotted in Figure 2 ( 28 and 29). Rabbit 29 (Figure 2) showed a greater neuronal response to the CS vs. the generalization tone in Extinction $1(p<.005)$, Extinction $2(p<.005)$, and Extinction $4(p<.01)$. The greatest effect occurred for this subject at a latency of $25 \mathrm{msec}$ (fourth abscissa point from left). The generalization stimulus produced a greater response in pretraining than the $\mathrm{CS}+$, by $5.8 \mathrm{SDs}$. However, the $\mathrm{CS}+$ produced a greater response than the generalization stimulus in Extinction Sessions 2 (5.3 SDs) and 4 (6.0 SDs) and an approximately equal response in Session 1 . The average difference between the CS-related responses vs. the generalized responses over the three extinction sessions shown in Figure 2 was $3.6 \mathrm{SDs}^{3}$

The two additional subjects yielding significant stimulus control effects in extinction ( 24 and 28) produced results which were unlike those of rabbit 29 . In each case, the generalization stimulus produced a response of significantly greater magnitude than the response to the $\mathrm{CS}$ in pretraining. The stimulus control effect resulted from an increase from pretraining to extinction in the response to the $\mathrm{CS}$, and no change in response to the generalization stimulus. Thus the response to the CS approximated the large magnitude of the response to the generalization stimulus which occurred in pretraining and in testing to the generalization stimulus. This effect is illustrated for one of these subjects (28) in Figure 2. It occurred in the first session of extinction. Note that there was a progressive decline in the magnitude of response to both stimuli over sessions of extinction for this subject.

The remaining subjects of Experiment $1(7,18$, 25 , and 35 ) yielded neuronal data which did not show stimulus control. Rather, the response profiles of these subjects remained constant in form over the stages of the experiment (e.g., rabbit 35, Figure 2).

Discussion. Four of the rabbits of Experiment 1 produced neuronal activity from the MGN which exhibited constancy over the stages of the experiment. In these cases, each stimulus produced a unique response profile which was maintained throughout the experiment. It would appear from these data that the MGN exhibits constant neuronal responsiveness reflecting the constant acoustic properties of the input. These results are in conformity with the traditional "static" view of the sensory systems.

Three of the rabbits yielded data suggesting associative change, in the form of stimulus control, within MGN. However, only one of these (29) provided a relatively unambiguous instance of associative change. The remaining two subjects showed differential neuronal responses favoring the generalization stimulus prior to conditioning. In both cases, the CS produced a very minimal response prior to conditioning. The stimulus control effect was produced by increase from pretraining to extinction 


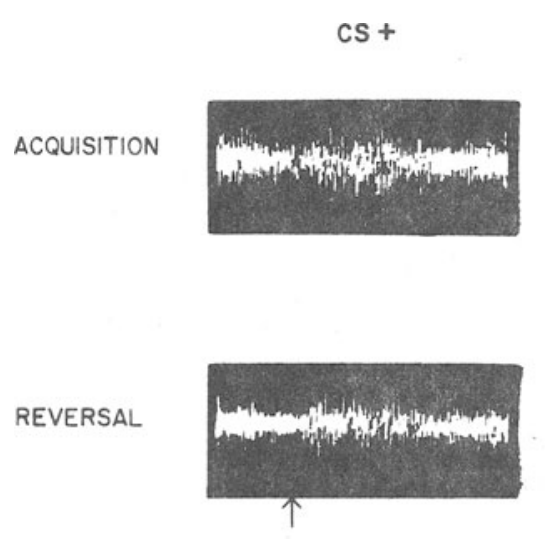

cs +
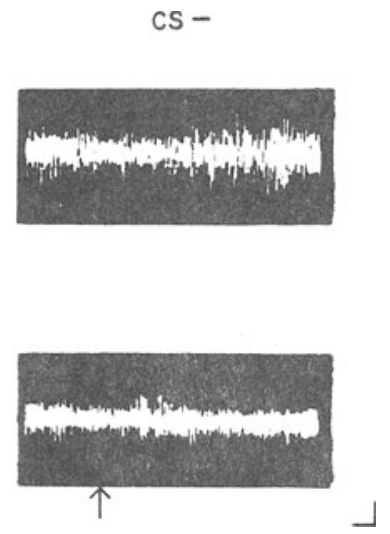

Figure 3. This figure depicts multiple-unit activity from the MGN of rabbit 44. Each individual photograph spans 120 msec beginning 30 msec prior to tone onset (arrows). Representative CS + trials of terminal acquisition and terminal reversal are shown in the left column. Photographs in the right column are of the first $\mathrm{CS}$ - trial which occurred after the $\mathrm{CS}+$ trial shown in that row. This figure demonstrates that the MGN discrimination effect shown by the histograms (Figure 4) may be seen on individual trials. In conditioning, virtually every stimulus pair $(\mathrm{CS}+, \mathrm{CS}-)$ showed the effect. In reversal, the majority of pairs showed the reverse discrimination. The horizontal calibration is $10 \mathrm{msec}$ and the vertical is $20 \mu \mathrm{V}$. in CS-related neuronal activity, relative to absence of change in the ample response to the generalization stimulus. The ambiguity here resided in the possibility that this outcome may have been produced by shock or arousal-related overall increase in the MGN response (see Buchwald, Halas, \& Schramm, 1966; Hall \& Mark, 1967), coupled with a possible "ceiling" effect limiting the amount of increase in the large pretraining response to the generalization stimulus. This alternative obviated a clear associative interpretation of the data of the two subjects involved. Thus, Experiment 1 produced marginal evidence suggesting associative change within the MGN. The evidence provided a rationale for further pursuit of the question.

The second experiment was designed in light of the outcome of Experiment 1. It involved an attempt to demonstrate stimulus control in MGN using the procedures of differential conditioning, followed by reversal within the same subject. The observation of neuronal reversal removes the ambiguities which arise from the observation of a "one-way" differential response.

\section{Experiment 2}

Five of the seven subjects who received differential conditioning and reversal showed significant crossover $^{4}$ from terminal acquisition to terminal reversal of the short-latency $(5-35 \mathrm{msec})$ MGN neuronal response. In other words, MGN neurons showed a differential response favoring the $\mathrm{CS}+$ at the end of acquisition. Just as the behavior, the MGN neuronal response reversed when the $\mathrm{CS}+$ and $\mathrm{CS}-$ were interchanged. Figure 3 illustrates the reversal effect by showing photographically the discriminative response in terminal acquisition and in terminal reversal for one rabbit (44). Neuronal crossover from terminal acquisition to terminal reversal is also shown for three rabbits in the rightmost segments of Figure $4(40,44,45)$. Additional instances of this effect are shown in a preliminary report of these data (Gabriel, Saltwick, \& Miller, 1975).

Reversal constitutes a contingency-specific modifi- cation of MGN activity. It cannot reasonably be attributed to nonassociative change accompanying conditioning. Moreover, reversal precluded the ambiguities of Experimènt 1. Thus, the data of Experiment 2 provided a clear demonstration of associative modification of primary sensory activity.

In order to clarify the relationship between MGN neuronal activity and the behavioral CR, plots of neuronal and behavioral data were juxtaposed for each of the five rabbits who showed the reversal effect (Figure 5). The lower portions of the graphs show the behavioral responses to $\mathrm{CS}+$ and $\mathrm{CS}-$ over sessions. The upper portions of the graphs show the magnitude of the differential neuronal response over sessions. Significant single-bin differential responses (i.e., difference scores $>|2.8|$ ) occurred relative to pretraining in acquisition and in reversal in each of the five rabbits.

An interesting aspect of the data shown in Figure 5 was the pattern of neuronal response observed over sessions of reversal. Unlike behavioral reversal, the onset of which occurred after several sessions in all rabbits, neuronal reversal occurred abruptly in the initial sessions in four of the rabbits. In three rabbits, there was a return to the differential neuronal response appropriate to original conditioning, followed by a second neuronal reversal in the terminal sessions (Figure 5, 41 and 45). One rabbit showed abrupt early reversal which was maintained throughout the remaining sessions (Figure 5, 40). Finally, one rabbit showed a neuronal response appropriate to original acquisition through most of reversal. Neuronal reversal did not occur for this animal until the last four sessions (Figure 5, 44).

In general, the neuronal and the behavioral data were in correspondence in the terminal sessions of acquisition and reversal; however, they were not in agreement during the initial and intermediate sessions of reversal. While the behavioral reversal required several sessions, MGN activity reversed abruptly, shifted back to the pattern observed in original conditioning in some subjects, 
maintained the reversal pattern in others, and showed reversal in the terminal sessions of all subjects.

The scores portrayed in Figure 5 were corrected for neuronal preference effects in pretraining. Since these scores showed a significant differential conditioning effect and a significant reversal effect, neither of these effects may be attributed to pretraining preferences. Nevertheless, the data did indicate that the acoustic properties of the stimuli influenced the neuronal activity of MGN. This point is illustrated by Figure 4 ( 45 and 40 ), which shows the $\mathrm{CS}+$ and $\mathrm{CS}-$ profiles of two rabbits in pretraining, three stages of conditioning (first, middle, and last sessions), and three stages of reversal. Note the greater magnitudes of the neuronal response to the $8-\mathrm{kHz}$ tone in the pretraining of rabbit 40 , and the greater response to the $1-\mathrm{kHz}$ tone in the pretraining of rabbit 45 .

Inspection of Figure 4 revealed an interesting aspect of the MGN profiles of terminal acquisition and of terminal reversal. The differential effect in reversal had a shorter latency than the effect in acquisition. This difference held in four of the five subjects which showed a reversal effect. The difference may have been related to the pretraining differences described above in neuronal response to the two tones, since by chance, the tone producing the lesser response in pretraining was the $\mathrm{CS}+$ in acquisition for all four of the rabbits. Thus, the short latency of the neuronal responses observed in reversal may have been related to the "preferred" nature of the $\mathrm{CS}+$ in reversal. In line with this interpretation is the fact that in one instance MGN activity did not show a stimulus preference in pretraining; moreover, the same activity did not show a difference in latency between acquisition and reversal (see Figure 4, 44).

Two rabbits did not show differential conditioning and reversal in MGN. One of these (50) exhibited significant neuronal discrimination appropriate to reversal, but not to original conditioning, relative to pretraining. The other (46) showed a constant differential effect throughout pretraining, differential conditioning, and reversal. Thus the results of Experiment 2 were similar to those of Experi-

Figure 4. This figure shows the MGN response of three rabbits, over the stages of Experiment 2. Each section (i.e., 45, 40, 44) is based on the MGN neuronal activity of a single rabbit. The subject number and $\mathrm{CS}+$ frequency for acquisition is shown in the lower left cell. Each individual graph is in the format of Figure 2. Response curves are presented for noncontingent shock pretraining (PT), differential conditioning (ACQ; first day, middle day, final day), and reversal (REV: first day, middle day, final day). The session number is indicated in the upper left of each graph. Rabbit 45 attained criterion for differential conditioning on the second day. Thus, only 2 days of differential conditioning are shown.

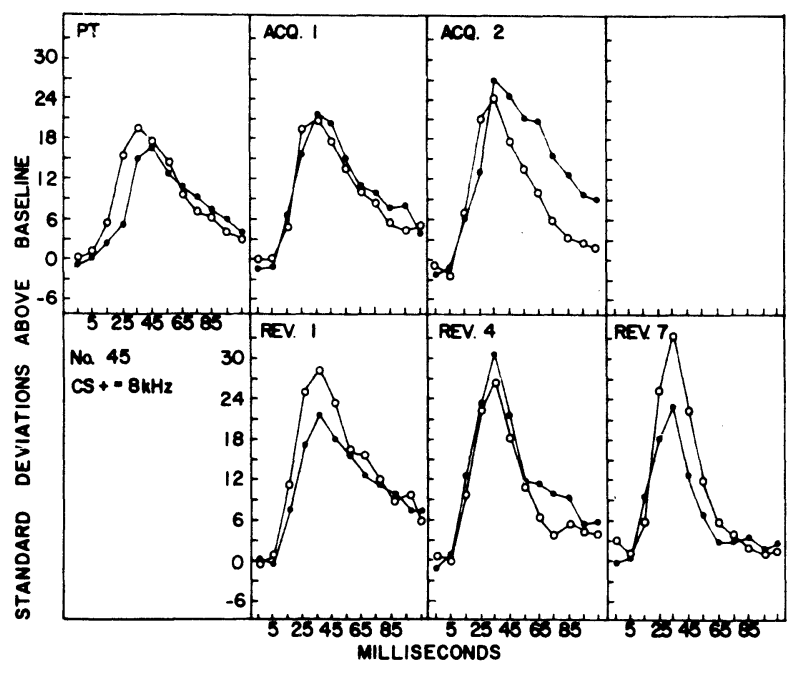

B
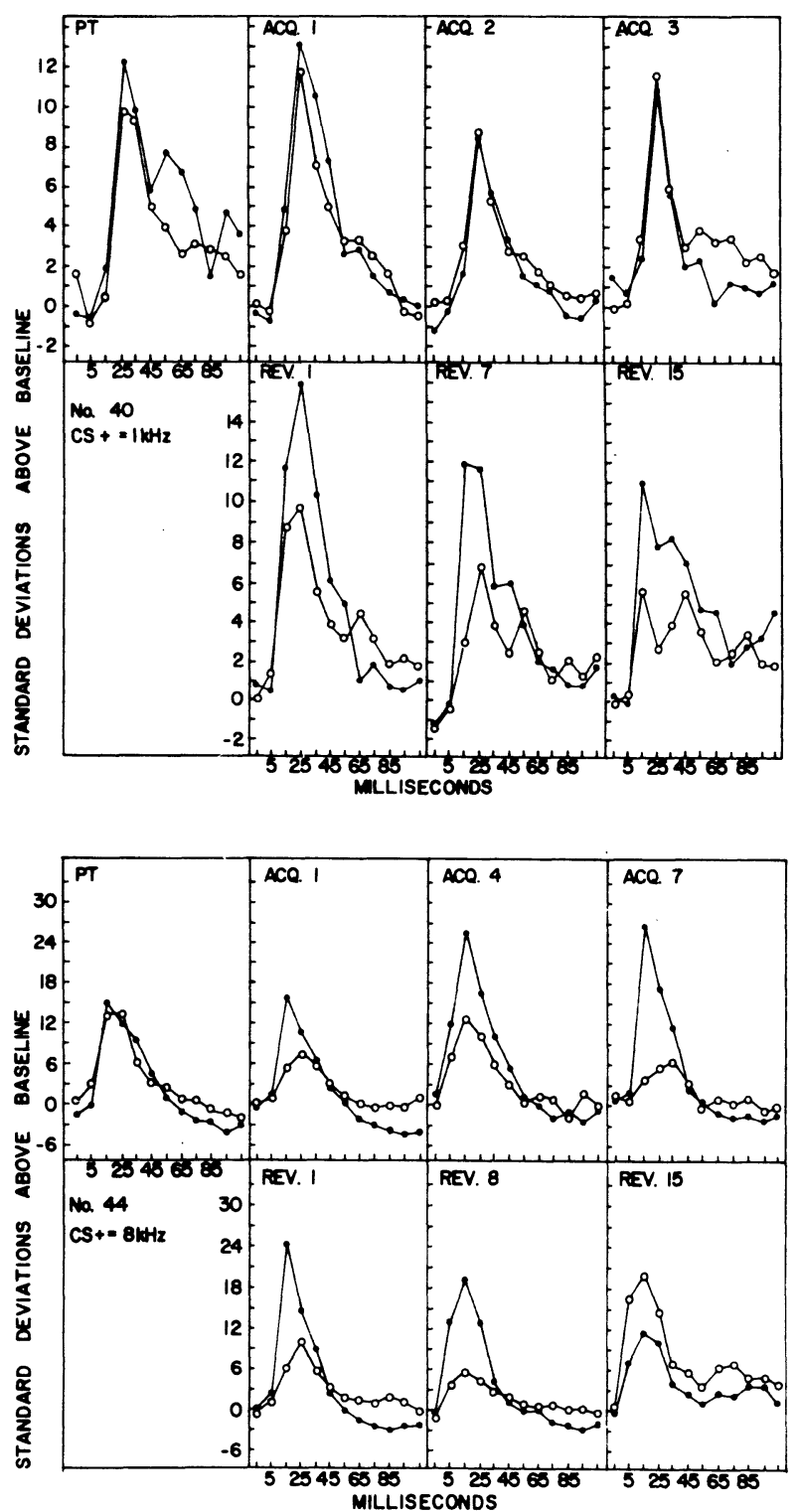
Figure 5. This figure shows the relationships between neuronal (MGN) and behavioral responses during differential conditioning and reversal. The lower portion of each graph shows the percentage of behavioral responses to the $\mathrm{CS}+$ and the $\mathrm{CS}-$ over consecutive sessions. The $\mathrm{CS}+$ of differential conditioning was an 8-kHz tone $(\theta)$ for two of the graphs shown (Nos. 45 and 44) and $a 1-k H z$ tone $(O)$ for the remaining graphs (Nos. 41, 42, 40). The upper portion of the graph shows the magnitude of the MGN differential response [CS + (acquisition) - CS - (acquisition)] in each session. The scores were based on the single 10-msec period (bin) after CS onset which showed the greatest crossover in terminal reversal relative to terminal acquisition. The same bin (Bins 3, $5,5,3,6$ for rabbits $40,41,42,44,45$, respectively) was used throughout the analysis of a given subject's data. ${ }^{5}$. Each bar shows the magnitude of the differential response (CS + - CS -) minus the magnitude of this difference obtained during noncontingent pretraining. Bars above the baseline in acquisition reflect a greater response to the acquisition $\mathrm{CS}+$ than to the acquisition CS - . Bars below the baseline in reversal indicate a greater response to the reversal CS + than to the reversal CS - . Long extinction was given between Sessions 5 and 6 for rabbits 41 and 42 , and between Sessions 10 and 11 for rabbit 41 . Rabbit $\mathbf{4 0}$ did not receive long extinction. Due to equipment problems, neuronal data were not obtained for Reversal Session 6 rabbit 42 or Reversal Session 5 rabbit 44.
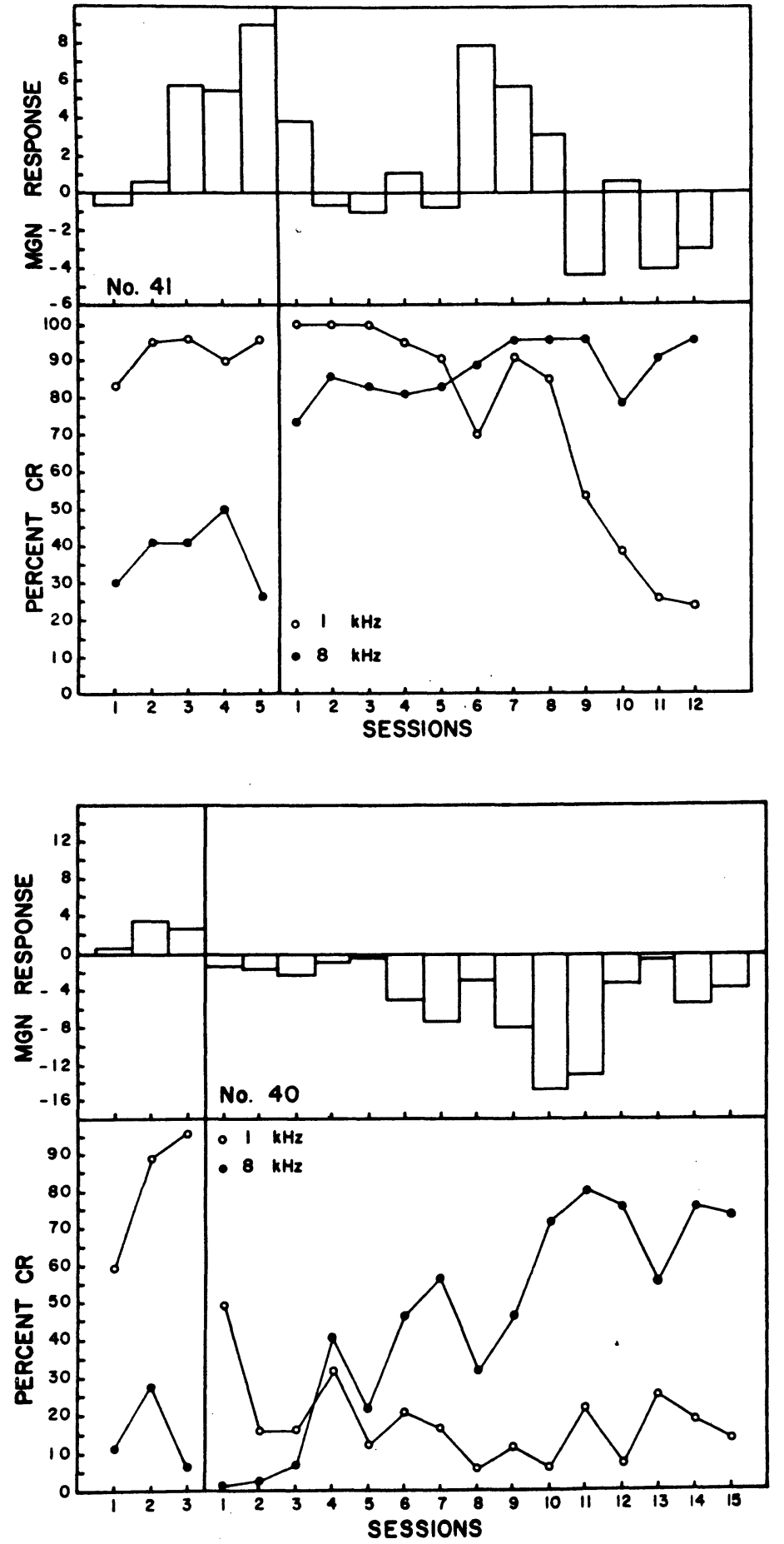

ment 1 in suggesting that associative modification occurs in terms of a limited subset of the regions in MGN. Moreover, the outcome for rabbit 50 suggested that some regions may exhibit tone-specific activity, with limited or partial potential for associative change.

Long-latency neuronal activity. The data of the second $100 \mathrm{msec}$, in the form of $\mathrm{t}$ scores (see methods, p. 125) indicated associative activity in both experi- ments. In Experiment 1, all but one subject (28) showed stimulus control in terms of $t$ scores corrected for pretraining differences. In Experiment 2, all subjects except 46 and 40 showed a significant amount $(t>2.10)$ of crossover. The amounts of change in $t$ units were $10.20,6.80,2.60,23.40$, and 6.10 for rabbits $41,42,44,45$, and 50 , respectively. Thus, with one exception (40), subjects who showed clear associative change in the first $100-\mathrm{msec}$ period 

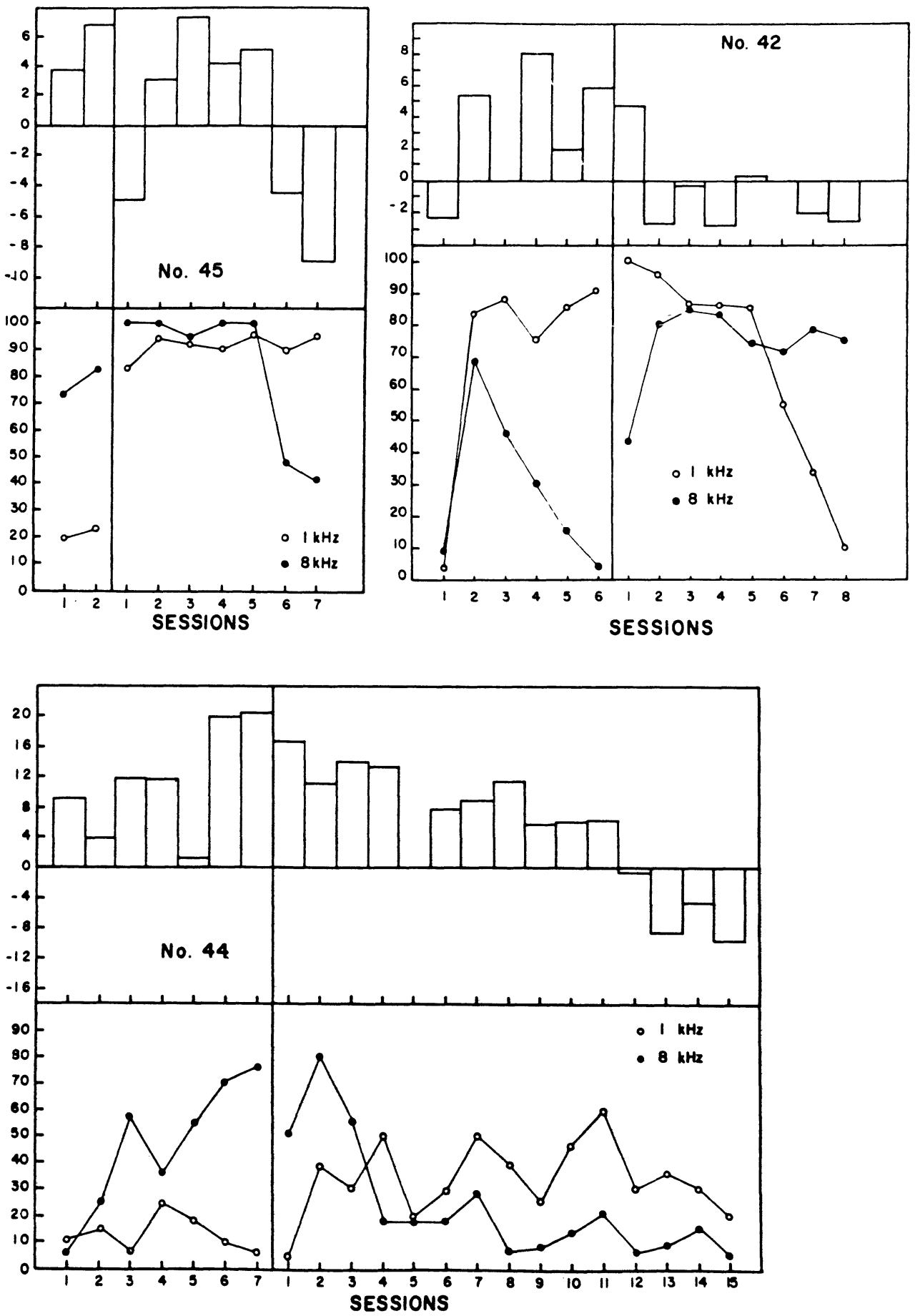

showed it in the second period. Also, several subjects not showing clear change in the first period $(7,18,25,35,50)$ did show change in the second period.

Histology. The positions of the electrodes in both experiments are shown in Figure 6. Both associative and nonassociative placements occurred along the full anterior-posterior extent of the nucleus. However, all of the placements which showed associ- ative activity were in the medial one-half of the nucleus, whereas only two of the six nonassociative placements were in the medial one-half. Thus, it may be that nonassociative neuronal activity occurs throughout the MGN, whereas a mediolateral organization of the MGN may exist with respect to associative activity. This suggestion is compatible with recent studies indicating a mediolateral organization of the MGN in terms of neuroanatomical (e.g., 


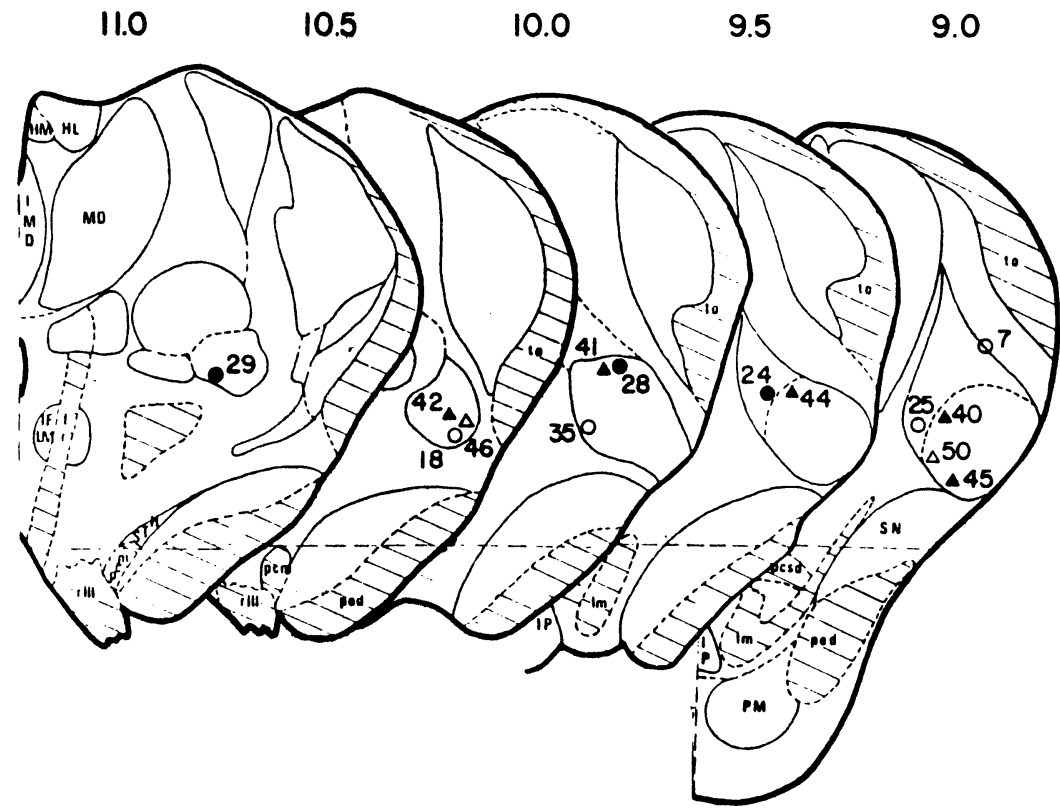

Figure 6. This figure illustrates the locations of the electrode tips for rabbits in both experiments. Drawings of the thalamus are taken from the atlas of Urban and Richard (1972). The distance in millimeters anterior to stereotaxic zero is shown above each section. Each placement is shown on the atlas plane that best approximates the location of the electrode tip. Circles denote placements in rabbits of Experiment 1 and triangles denote placements in rabbits of Experiment 2. Filled symbols denote placements yielding shofi-latency associative neuronal activity (see p. 000). The rabbit number for each placement is adjacent to the symbol for that placement.

Morest, 1964) and neurophysiological (e.g., Aitkin, 1973; Love \& Scott, 1969) properties.

\section{DISCUSSION}

The primary finding of these studies was differential conditioning and reversal of short-latency neuronal activity of the rabbit medial geniculate nucleus. This outcome supported the idea that activity in the afferent pathways is altered by change in the associative significance of incoming stimuli. The data are in opposition to the traditional view that sensory neuronal activity serve only to "represent" stimuli within the brain.

Certain studies similar to the present one (e.g., Hernández-Peón, Scherrer, \& Jouvet, 1956) have suffered severe criticism because the differential neuronal activity which was reported may have been produced by differential body and/or receptor orientation, rather than by change intrinsic to the CNS. However, orientation cannot account for the present data, because the two stimuli occurred in a randomized sequence and there was no basis for the subject to "predict" which would occur, and thus to orient differentially. Moreover, it is unlikely that the effects could be accounted for in terms of a tonic set of the middle-ear muscles. Studies using a variety of species have shown selective suppression, due to the middle-ear mechanism of transmission of auditory frequencies below $2 \mathrm{kHz}$ (Mфller, 1972). However, the same studies have also indicated that the middle-ear mechanism does not mediate suppression of frequencies above $2 \mathrm{kHz}$. Thus, the middle-ear mechanism does not seem to be adequate to acrount for the instances in our data of sup- pression to the $8-\mathrm{kHz}$ tone (e.g., terminal reversal, Figure 4, 44). Moreover, no evidence is available suggesting that the middle-ear mechanism may mediate modifiable selectivity of auditory transmission as would be required by our reversal effect. It may be true, however, that the effect seen in the present study was mediated via a neural structure of the auditory system more peripheral than MGN. In fact, a very recent study (Oleson, Ashe, \& Weinberger, 1975) has shown long-latency associative changes to occur at the cochlear nucleus. Because of their latency, these alterations may have been secondary to associative changes occurring elsewhere in the nervous system. Nevertheless, they suggest the possibility of farperipheral associative selectivity in the auditory system.

It is interesting to consider the neural mechanism underlying neuronal differential conditioning and reversal. On the one hand, it is possible that the activity resulted from a biasing of neurons, which was acquired during conditioning. The biasing may have been mediated by way of a tonic flow of impulses along a centrifugal neural pathway, such as the corticofugal pathways to MGN demonstrated by Pontes, Reis, \& Sousa-Pinto (1975) and Watanabe et al. (1966). Such tonic neural input may contain sufficient informational specificity to reduce activity evoked by the CS - and/or to enhance activity evoked by the $\mathrm{CS}+$. This hypothetical mechanism represents a selective sensory filtering process of the kind envisioned by Hernández-Peón et al. (1956), and believed by him to underlie the phenomena of selective attention. On the other hand, it is possible that the associative activity resulted from an alteration of the structure of the neurons in MGN. Perhaps 
an "engram" comes to reside in MGN as a result of conditioning. This alternative is the one stated by Olds and his colleagues (Olds, Disterhoft, Segal, Kornblith, \& Hirsh, 1972) to account for shortlatency neuronal activity which they observed to accompany conditioning in rats.

The present data would seem to favor the former of these hypotheses. This is indicated by the abrupt shifts which occurred between the differential neuronal response appropriate to original conditioning and the response appropriate to reversal. It is more reasonable to account for such shifts in terms of a rapid switching of the pattern of centrifugal flow into MGN than in terms of a rapid manufacture, dissolution, and remanufacture of engrammic structures. This is especially true in light of the observation that the MGN effect is in all likelihood a context-dependent effect which takes on a different set of properties in each situation encountered by the animal. The MGN set appropriate to a given context is most probably established via several of the sensory modalities. By this account, neural influences external to MGN must govern its stimulus-filtering properties.

Whether our effects were mediated by a biasproducing centrifugal flow or by an engram localized within MGN, it would seem more reasonable to view them generally as phenomena of stimulus selection acquired through learning, rather than neural changes underlying the learning of the behavioral response. This assertion is based on the absence of a close relationship during reversal between MGN and the behavioral indices of discrimination. Recall that neuronal reversal occurred in some rabbits on the first day of reversal training, whereas behavioral reversal always required several sessions. However, one subject (Figure 4, 44) reversed behaviorally on Session 4 of reversal training, but did not show MGN reversal until Session 11. Caution should be used in drawing inferences from this single outcome, in that other circumstances were somewhat unusual. Although this subject showed statistically reliable behavioral discrimination during reversal training, he never attained our criterion of reversal. Nevertheless, the overall importance of these data seems to be as mentioned, that a very close tie did not exist between MGN and behavioral discrimination. Thus it does not seem that MGN activity is a necessary precursor of behavioral responding. This conclusion is similar to that of Oleson, Ashe, and Weinberger (1975), who maintained that auditory neuronal changes did not form the neural basis of their behavioral (pupillary) conditioning effect. The conclusion is also compatible with the hypothesis that the MGN effect reflected a sensory filtering mechanism.

Under ecologically normal circumstances, sensory filtering would be assumed to be acquired in parallel with behavioral learning. Our own initial discrimina- tion task, presumed to be fundamentally similar to possible discriminative problems which may occur in the natural environment, illustrates such acquisition. That is, during original conditioning, neuronal and behavioral discrimination occurred in parallel. There was no clear precedence of one effect over the other. In this situation, selective filtering would facilitate appropriate behavior by emphasizing relevant stimuli and/or by deemphasizing irrelevant ones. However, under unusual circumstances rarely encountered in a natural environment (reversal), it is possible to show the independence of MGN sensory filtering and behavioral discrimination, as in the case of the shifting, during reversal, of MGN activity between the acquisition and reversal patterns.

It is interesting to consider the nature of the cellular events which gave rise to the present associative effects. On the one hand, it is possible that the activity of single cells within the MGN undergoes differential conditioning and that the activity of the same cells crosses over in reversal. Alternatively, it may be that most cells of MGN show a "prewired" response favoring a particular range of stimuli. Differential conditioning may produce suppression of CS-related activity of cells whose favored stimulus is, or is similar to, the CS-, and enhancement of activity of cells whose favored stimulus is, or is similar to, the CS +. Under this alternative, the individual cells retain their favored stimuli but show an increased response when a favored stimulus is $\mathrm{CS}+$ and a decreased response when a favored stimulus is CS - . The first of these alternatives implies that the associative MGN response derives from modification of the activity of individual cells, whereas the second alternative involves alteration of the activity of intermixed subpopulations of neurons with fixed response properties.

The question is which of these alternatives is correct? To answer this, it is necessary to maintain individual cellular recordings throughout the course of differential conditioning and reversal. Only in this way would it be possible to determine whether the same single neurons show differential activity appropriate both to conditioning and to reversal.

\section{REFERENCE NOTE}

1. Gabriel, M., Saltwick, S. E., \& Miller, J. D. Limbic and auditory neuronal response to tones with and without noncontingent shock. In preparation, 1975.

\section{REFERENCES}

Aitkin, L. M. Medial geniculate body of the cat: Responses to tonal stimuli of neurons in the medial division. Journal of Neurophysiology, 1973, 36, 275-282.

Ammassian, V. E. Studies on organization of a somesthetic association area, including a single unit analysis. Journal of Neurophysiology, 1954, 17, 39-58.

Berlucci, G., Munson, J. B., \& Rizzolatti, G. Changes in click-evoked responses in the auditory system and the 
cerebellum of free-moving cats during sleep and waking. Archives Italiennes de Biologie, 1967, 105, 118-135.

Broadbent, D. E., \& Gregory, M. Stimulus set and response set: The alternation of attention. Quarterly Joumal of Experimental Psychology, 1964, 16, 309-312.

Brogden, W. J., \& Culler, E. A. Device for motor conditioning of small animals. Science, 1936, 83, 269.

Buchwald, J. S., Halas, E. S., \& Schramm, S. Changes in cortical and sub-cortical unit activity during behavioral conditioning. Physiology and Behavior, 1966, 1, 11-22.

Dunlop, C. W., Webster, W. R., \& Simons, L. A. Effect of attention on evoked responses in the classical auditory pathway. Nature, 1965, 206, 1048-1050.

Fox, C. A., \& Eichman, J. A rapid method for locating intracerebral electrode tracks. Stain Technology, 1959, 24, 39-42.

GABRIEL, M. Effects of intersession delay and training level in avoidance extinction and intertrial behavior. Journal of Comparative and Physiological Psychology, 1968, 66, 412-416.

GABRIEL, M. A system for multiple-unit recording during avoidance behavior of the rabbit. Physiology and Behavior, 1973, 12, 145-148.

Gabriel, M., Saltick, S. E., \& Miller, J. D. Conditioning and reversal of short latency multiple-unit responses in the rabbit medial geniculate nucleus, Science, 1975, 189, 1108-1109.

Granit, R. Centrifugal and antidromic effects on ganglion cells of retina. Journal of Neurophysiology, 1955, 18, 388-411.

HALl, R. D., \& MARx, R. G. Fear and modification of acoustically evoked potentials during conditioning. Journal of Neurophysiology, 1967, 30, 893-910.

Hernández-Peón, R., Scherrer, H., \& Jouvet, M. Modification of electrical activity in cochlear nucleus during "attention" in unanesthetized cats. Science, 1956, 123, 311-332.

HoRN, G., \& HrL, R. M. Habituation of the response to sensory stimuli of neurons in the brain stem of rabbits. Nature, 1964, 202, 296-298.

Hubel, D. H., \& Wiesel, T. N. Receptive fields of single neurons in the cat's striate cortex. Journal of Physiology, 1959, 148, 574-591.

Hutrenlocher, P. R. Evoked and spontaneous activity in single units of medial brain stem during natural sleep and waking. Journal of Neurophysiology, 1961, 24, 451-468.

Imig, T. J., \& WeInberger, N. M. Relationships between rate and pattern of unitary discharges in MGN of the cat in response to click and amplitude-modulated white noise stimulation. Journal of Neurophysiology, 1973, 36, 385-398.

Love, J. A., \& Scorr, J. W. Some response characteristics of cells of the magnocellular division of the medial geniculate body of the cat. Canadian Journal of Physiology and Pharmacology, 1969, 47, 881-888.

Møller, A. R. The middle ear. In J. V. Tobias (Ed.), Foundations of modern auditory theory. New York: Academic Press, 1972.

Morest, D. K. The neuronal architecture of the medial geniculate body of the cat. Journal of Anatomy, 1964, 98, 611-630.

Mori, K., Winters, W. D., \& Spooner, C. E. Comparison of reticular and cochlear multiple unit activity with auditory evoked responses during various stages induced by anesthetic agents. Electroencephalography and Clinical Neurophysiology, $1968,24,242-248$.

Olds, J., Disterhoft, J., Segal, M., Kornblith, D. L., \& HIRSH, R. Learning centers of rat brain mapped by measuring latencies of conditioned unit responses. Journal of Neurophysiology, 1972, 35, 202-219.

Oreson, T. D., Ashe, J. H., \& Weinberger, N. W. Modification of auditory and somatosensory system activity during pupillary conditioning in the paralysed cat. Journal of Neurophysiology, 1975, 38, 1114-1139.

Pontes, C., Reis, F. F., \& Sousa-Pinto, A. The auditory cortical projections onto the medial geniculate body in the cat: An experimental anatomical study with silver and autoradiographic methods. Brain Research, 1975, 91, 43-63.

Price, G. R. Middle ear muscle activity in the rabbit. I. Loss of threshold. Journal of Auditory Research, 1963, 3, 221-231.

Rescorla, R. A. Pavlovian conditioning and its proper control procedures. Psychological Review, 1967, 74, 71-80.

SIEGEL, S. Nonparametric statistics for the behavioral sciences. New York: McGraw-Hill, 1956.

Simons, L. A., Dunlop, C. W., Webster, W. R., \& Aitkin, L. M. Acoustic habituation in cats as a function of stimulus rate and the role of temporal conditioning of the middle ear muscles. Electroencephalography and Clinical Neurophysiology, $1966,20,485-493$.

Teas, D. C., \& Kiang, N. Y. S. Evoked responses from the auditory cortex. Experimental Neurology, 1964, 10, 81-118.

Treisman, A. M. Contextual cues in selective listening. Quarterly Journal of Experimental Psychology, 1960, 12, 242-248.

Thompson, R. F., \& Spencer, W. A. Habituation: A model phenomenon for the study of neuronal substrates of behavior. Psychological Review, 1966, 173, 16-43.

URBAN, I., \& Richard, P. A stereotaxic atlas of the New Zealand rabbit's brain. Springfield, Ill: Thomas, 1972.

Watanabe, T., Yanagisawa, K., Kanzaki, J., \& Katsuki, Y. Cortical efferent flow influencing unit responses of medial geniculate body to sound stimulation. Experimental Brain Research, 1966, 2, 302-317.

Webster, W. R. The effects of repetitive stimulation on auditory evoked potentials. Electroencephalography and Clinical Neurophysiology, 1971, 30, 318-330.

WICKELGREN, W. O. Effect of state of arousal on click evoked responses in cat. Joumal of Neurophysiology, 1968, 31, 757-768.

WINTER, D. L. N. Gracilis of cat: Functional organization and corticofugal effects. Joumal of Neurophysiology. 1965. 28. 48-70.

\section{Notes}

1. During overtraining the intensity of the CS was varied over four values: $70,75,80,85,90 \mathrm{~dB}$. These values occurred in a random sequence with each intensity presented equally often in a session.

2. Rabbits who did not attain criterion in five consecutive sessions were given "long extinction" on the sixth session. In long extinction, 240 presentations of the CS - were given without shock. Long extinction sessions were continued on consecutive days, if required, until a criterion of $19 / 20$ nonresponses occurred in any 20-trial block. This procedure hastened differential conditioning and/or reversal in slow learners. Long extinction was required for only one subject in differential conditioning but $60 \%$ of the subjects required long extinction in reversal.

3. The associative intepretation of these data was strengthened by the observation of development in this subject of a significant differential neuronal effect during differential conditioning, carried out after the present study. The generalization stimulus of the present study was used as the CS + and the CS + became CS - so that the differential effect, in fact, represented a "reversal" of the stimulus control effect obtained here.

4. Amount of crossover was defined as $[\mathrm{Z}(1 \mathrm{kHz})-(\mathrm{Z} 8 \mathrm{kHz})$, terminal acquisition $]-[\mathrm{Z}(1 \mathrm{kHz})-\mathrm{Z}(8 \mathrm{kHz})$, terminal reversal]. This expression would have an expected value of zero if the MGN response were purely tone-specific. However, reversal of the scores or sufficient change approximating reversal yields a nonzero (statistically significant) expected outcome.

5. Essentially the same results were obtained when the average scores from five consecutive bins were used instead of a single bin. The five bins used were the single bin showing the greatest crossover plus two bins on either "side" of the single bin.

(Received for publication August 1975; revision received December 1975.) 